\title{
A New Numerical Procedure to Compute the Residues of a Complex Functions
}

\author{
Abbas Y. Al-Bayati \\ profabbasalbayati@yahoo.com \\ College of Computer Sciences \\ and Mathematics \\ University of Mosul/Iraq
}

Received on: $27 / 08 / 2006$

\author{
Sasan A. Al-Shwani \\ College of Science \\ University of Kirkuk
}

Accepted on: 16/11/2006

\section{ABSTRACT}

In this paper, we are going to deal with computations of Residues and Poles for the complex functions . We are also going to investigate a new numerical procedure theoretically and its implementation numerically to compute the residue of complex analytic functions with high order poles. The paper needs the knowledge of computing the complex improper integrations.

Keywords: complex analytic functions, Residues and Poles, complex improper integrations.

$$
\begin{aligned}
& \text { طريقة عددية لحساب الرواسب والأقطاب في الدوال العقدية } \\
& \text { عباس يونس البياتي } \\
& \text { ساسان عادل الثواني } \\
& \text { كلية علوم الحاسوب والرياضيات/جامعة الموصل يونسي الياتي } \\
& \text { جامعة كركوك/كلية العلوم } \\
& \text { تاريخ قبول البحث: 2006/11/16 } \\
& \text { تاريخ استلام البحث: 2006/8/27 }
\end{aligned}
$$

\section{1-Introduction :}

A final bit of theory in this paper is devoted to Cauchy's residue theorem and its applications . Recall that Cauchy's theorem stated that $(\oint f(z) d z=0)$ so long as $\mathrm{f}(\mathrm{z})$ was analytic every where inside C.What if $\mathrm{f}(\mathrm{z})$ is not analytic within $C$ ?

The answer is provided by Cauchy's integral formula. Here we have proposed a new procedure to compute the complex improper integrations by calculating the residues of the function . 
To compute the residues of a complex function of the form $\left(f(z)=\frac{p(z)}{q(z)}\right)$ such that the function $\mathrm{f}(\mathrm{z})$ has a pole at $\left(z=z_{o}\right)$, we are going to use the (short - cut - method) to estimate the residues[9].

If $\mathrm{R}$ is the real field and $\mathrm{C}$ is the complex field then consider the following definitions and theorems:

\section{1-1 poles :[3]}

If we find a positive integer $(\mathrm{m})$ such that $\left[\lim _{z \rightarrow z_{o}}\left(z-z_{o}\right)^{m} f(z) \neq 0\right]$ Then $\left(z=z_{o}\right)$ is called a pole of order $(\mathrm{m})$. If $(\mathrm{m}=1) \quad\left(z_{o}\right)$ is called a simple pole.

\section{1-2 Analytic functions :[9]}

If the derivative $f^{\prime}(z)$ exists at all points $(\mathbf{z})$ of a region $\mathbf{R}$, then $f(z)$ is said to be analytic in R.

\section{1-3 Taylor's theorem :[3]}

Let $\mathrm{f}(\mathrm{z})$ be analytic inside and on a simple closed curve $\mathrm{C}$. Let (a) and $(\mathrm{a}+\mathrm{h})$ be two points inside $\mathrm{C}$, then

$$
\begin{aligned}
& f(a+h)=f(a)+h f^{\prime}(a)+\frac{h^{2}}{2 !} f^{\prime \prime}(a)+\ldots . . \quad \text { or writing as } \\
& f(z)=f(a)+(z-a) f^{\prime}(a)+\frac{(z-a)^{2}}{2 !} f^{\prime \prime}(a)+\ldots . .
\end{aligned}
$$

Where $(\mathrm{z}=\mathrm{a}+\mathrm{h})$

The above series is called Taylor expansion for $\mathrm{f}(\mathrm{z})$.

\section{1-4 Cauchy integral formula :[2]}

If $\mathrm{f}(\mathrm{z})$ is analytic inside and on a simple closed curve $\mathrm{C}$ and (a) is any point inside $\mathrm{C}$ then

$$
f(a)=\frac{1}{2 \pi i} \int_{c} \frac{f(z)}{(z-a)} d z
$$

Where $\mathrm{C}$ is traversed in the positive sense also the nth derivative of $(\mathrm{z}=\mathrm{a})$ is given by

$$
f^{(n)}(a)=\frac{1}{2 \pi i} \int_{c} \frac{f(z)}{(z-a)^{n+1}} d z \quad ; \quad \mathrm{n}=1,2,3, \ldots . .
$$

1-5 Lemma (1) :[9] 
Let $\left(Z_{o}\right)$ be a pole of order $(\mathrm{m})$ of a function $\mathrm{f}(\mathrm{z})$ then the residue of the function at a pole given by the form :

$$
\operatorname{Re} s\left(f, Z_{o}\right)=\frac{1}{(m-1) !} \lim _{z \rightarrow z_{o}} \frac{d^{m-1}}{d z^{m-1}}\left[\left(z-z_{o}\right)^{m} f(z)\right] .
$$

The formula namely (short - cut - method ) .

\section{2- A New method to compute the residues of a complex function:}

If $\mathrm{f}(\mathrm{z})$ has a pole $\left(z=z_{o}\right)$ of order $(\mathrm{m})$ then

$\frac{1}{2 \pi i} \oint f(z) d z=\frac{1}{(m-1) !} \lim _{z \rightarrow z_{o}} \frac{d^{m-1}}{d z^{m-1}}\left[\left(z-z_{o}\right)^{m} f(z)\right] \quad$ where $\quad\left[f(z)=\frac{p(z)}{q(z)}\right]$

We know that :

$$
\operatorname{Re} s\left(f, z_{o}\right)=\frac{1}{(m-1) !} \lim _{z \rightarrow z_{o}} \frac{d^{m-1}}{d z^{m-1}}\left[\left(z-z_{o}\right)^{m} f(z)\right]
$$

We find the value of the above integration by calculating the residues by this procedure and it can be found in [5].

2.1) If $\left(z=z_{o}\right)$ is a simple pole i.e $\left\lfloor q(z)=0, q^{\prime}\left(z_{o}\right) \neq 0\right\rfloor$ then the residue is given by the following form :

$$
\operatorname{Re} s\left(f, z_{o}\right)=\frac{p\left(z_{o}\right)}{q^{\prime}\left(z_{o}\right)}
$$

2.2) If $\left(z=z_{o}\right)$ is a pole of order $(\mathrm{m}=2)$ i.e $\left\lfloor q\left(z_{o}\right)=0, q^{\prime}\left(z_{o}\right)=0, q^{\prime \prime}\left(z_{o}\right) \neq 0\right\rfloor$ then the residue is given by the following form :

$$
\operatorname{Re} s\left(f, z_{o}\right)=2 \times \frac{p^{\prime}\left(z_{o}\right)}{q^{(2)}\left(z_{o}\right)}-\frac{2}{3} \times \frac{p\left(z_{o}\right) \cdot q^{(3)}\left(z_{o}\right)}{\left(q^{(2)}\left(z_{o}\right)\right)^{2}}
$$

See [5] for the details of these procedures.

\section{3- In this paper we are going to follow a procedure for a general} function of a pole of order $(\mathrm{m})$ :

3.1) Let us start with order $(\mathrm{m}=3)$ :

If $\left(z=z_{o}\right)$ is a pole of order $(\mathrm{m}=3)$ i.e

$\left\lfloor q\left(z_{o}\right)=0, q^{\prime}\left(z_{o}\right)=0, q^{\prime \prime}\left(z_{o}\right)=0, q^{\prime \prime \prime}\left(z_{o}\right) \neq 0\right\rfloor$ then the residue is given by the following form : 


$$
\begin{aligned}
\operatorname{Re} s\left(f, z_{o}\right)= & 3 \times\left[\frac{p^{(2)}\left(z_{o}\right)}{q^{(3)}\left(z_{o}\right)}-\frac{1}{10} \times \frac{p\left(z_{o}\right) \cdot q^{(5)}\left(z_{o}\right)}{\left(q^{(3)}\left(z_{o}\right)\right)^{2}}\right. \\
& \left.-\frac{1}{2} \times \frac{q^{(4)}\left(z_{o}\right) p^{(1)}\left(z_{o}\right)}{\left(q^{(3)}\left(z_{o}\right)\right)^{2}}+\frac{1}{8} \times \frac{\left(q^{(4)}\left(z_{o}\right)\right)^{2} p\left(z_{o}\right)}{\left(q^{(3)}\left(z_{o}\right)\right)^{3}}\right]
\end{aligned}
$$

\section{Proof:}

If $\left(z=z_{o}\right)$ is a pole of order $(\mathrm{m}=3)$ then by (short-cut-method) we get :

$\operatorname{Re} s\left(f, z_{o}\right)=\frac{1}{2 !} \lim _{z \rightarrow z_{o}} \frac{d^{2}}{d z^{2}}\left[\left(z-z_{o}\right)^{3} \frac{p(z)}{q(z)}\right]$

We expand the analytic function $\mathrm{q}(\mathrm{z})$ into Taylar series valid in disk

$\left|z-z_{o}\right|<r$

$$
\begin{aligned}
& =\frac{1}{2} \lim _{z \rightarrow z_{o}} \frac{d^{2}}{d z^{2}}\left[\frac{p(z)\left(z-z_{o}\right)^{3}}{q\left(z_{o}\right)+\left(z-z_{o}\right) q^{(1)}\left(z_{o}\right)+\frac{\left(z-z_{o}\right)^{2}}{2 !} q^{(2)}\left(z_{o}\right)+\ldots \ldots \ldots . .}\right] \\
& =3 \lim _{z \rightarrow z_{o}} \frac{d^{2}}{d z^{2}}\left[\frac{p(z)}{U(z)}\right]
\end{aligned}
$$

\section{Where}

$U(z)=q^{(3)}\left(z_{o}\right)+\frac{\left(z-z_{o}\right)}{4} q^{(4)}\left(z_{o}\right)+\frac{\left(z-z_{o}\right)^{2}}{5 \times 4} q^{(5)}\left(z_{o}\right)+\frac{\left(z-z_{o}\right)^{3}}{6 \times 5 \times 4} q^{(6)}\left(z_{o}\right)+\ldots$

Then from (6) we get :

$$
\begin{array}{r}
=3 \lim _{z \rightarrow z_{o}} \frac{d}{d z}\left[\frac{U(z) p^{(1)}(z)-p(z) U^{(1)}(z)}{(U(z))^{2}}\right] \\
=3 \lim _{z \rightarrow z_{o}}\left[\frac{U(z) p^{(2)}(z)-p^{(1)}(z) U^{(1)}(z)}{(U(z))^{2}}\right. \\
\left.-\frac{p(z) U^{(2)}(z)+U^{(1)}(z) p^{(1)}(z)}{(U(z))^{2}}-2 \times \frac{\left(U^{(1)}(z)\right)^{2} p(z)}{(U(z))^{3}}\right]
\end{array}
$$




$$
\begin{aligned}
& \lim _{z \rightarrow z_{o}} p(z)=p\left(z_{o}\right) \\
& \lim _{z \rightarrow z_{o}} p^{(1)}(z)=p^{(1)}\left(z_{o}\right) \\
& U(z)=q^{(3)}\left(z_{o}\right)+\frac{\left(z-z_{o}\right)}{4} q^{(4)}\left(z_{o}\right)+\frac{\left(z-z_{o}\right)^{2}}{5 \times 4} q^{(5)}\left(z_{o}\right)+\ldots \\
& \lim _{z \rightarrow z_{o}} U(z)=q^{(3)}\left(z_{o}\right) \\
& U^{(1)}(z)=\frac{1}{4} q^{(4)}\left(z_{o}\right)+\frac{2}{5 \times 4}\left(z-z_{o}\right) q^{(5)}\left(z_{o}\right)+\ldots \ldots \\
& \lim _{z \rightarrow z_{o}} U^{(1)}(z)=\frac{1}{4} q^{(4)}\left(z_{o}\right) \\
& U^{(2)}(z)=\frac{2}{5 \times 4} q^{(5)}\left(z_{o}\right)+\frac{3 \times 2}{6 \times 5 \times 4}\left(z-z_{o}\right) q^{(6)}\left(z_{o}\right)+\ldots \ldots . \\
& \lim _{z \rightarrow z_{o}} U^{(2)}(z)=\frac{1}{10} q^{(5)}\left(z_{o}\right)
\end{aligned}
$$

Then Substituting the above values in (10) in equation (9) we get the desired proof .

3.2) If $\left(z=z_{o}\right)$ is a pole of order $(\mathbf{m}=4)$ i.e $\left\lfloor q\left(z_{o}\right)=0, q^{\prime}\left(z_{o}\right)=0, q^{\prime \prime}\left(z_{o}\right)=0, q^{\prime \prime \prime}\left(z_{o}\right)=0, q^{\prime \prime \prime \prime}\left(z_{o}\right) \neq 0\right\rfloor$ then the residue is given by the following form :

$$
\begin{aligned}
\operatorname{Re} s\left(f, z_{o}\right) & =4 \times\left[\frac{p^{(3)}\left(z_{o}\right)}{q^{(4)}\left(z_{o}\right)}-\frac{3}{5} \times \frac{p^{(2)}\left(z_{o}\right) q^{(5)}\left(z_{o}\right)}{\left(q^{(4)}\left(z_{o}\right)\right)^{2}}\right. \\
& -\frac{1}{35} \times \frac{p\left(z_{o}\right) q^{(7)}\left(z_{o}\right)}{\left(q^{(4)}\left(z_{o}\right)\right)^{2}}-\frac{1}{5} \times \frac{p^{(1)}\left(z_{o}\right) q^{(6)}\left(z_{o}\right)}{\left(q^{(4)}\left(z_{o}\right)\right)^{2}} \\
& +\frac{2}{25} \times \frac{p\left(z_{o}\right) q^{(5)}\left(z_{o}\right) q^{(6)}\left(z_{o}\right)}{\left(q^{(4)}\left(z_{o}\right)\right)^{3}}+\frac{6}{25} \times \frac{\left(q^{(5)}\left(z_{o}\right)\right)^{2} p^{(1)}\left(z_{o}\right)}{\left(q^{(4)}\left(z_{o}\right)\right)^{3}} \\
& \left.-\frac{6}{125} \times \frac{\left(q^{(5)}\left(z_{o}\right)\right)^{3} p\left(z_{o}\right)}{\left(q^{(4)}\left(z_{o}\right)\right)^{4}}\right]
\end{aligned}
$$

\section{Proof:}

If $\left(z=z_{o}\right)$ is a pole of order $(\mathbf{m}=\mathbf{4})$ then by (short - cut - method) we get : 
$\operatorname{Re} s\left(f, z_{o}\right)=\frac{1}{3 !} \lim _{z \rightarrow z_{o}} \frac{d^{3}}{d z^{3}}\left[\left(z-z_{o}\right)^{4} \frac{p(z)}{q(z)}\right]$

We expand the analytic function $\mathrm{q}(\mathrm{z})$ into Taylor series valid in disk

$\left|z-z_{o}\right|<r$

$=\frac{1}{3 !} \lim _{z \rightarrow z_{o}} \frac{d^{3}}{d z^{3}}\left[\frac{\left(z-z_{o}\right)^{4} p(z)}{q\left(z_{o}\right)+\left(z-z_{o}\right) q^{(1)}\left(z_{o}\right)+\frac{\left(z-z_{o}\right)^{2}}{2 !} q^{(2)}\left(z_{o}\right)+\ldots . .}\right]$
$=4 \lim _{z \rightarrow z_{o}} \frac{d^{3}}{d z^{3}}\left[\frac{p(z)}{q(z)}\right]$

Where :

$U(z)=q^{(4)}\left(z_{o}\right)+\frac{\left(z-z_{o}\right)}{5} q^{(5)}\left(z_{o}\right)+\frac{\left(z-z_{o}\right)^{2}}{6 \times 5} q^{(6)}\left(z_{o}\right)+\ldots .$.

Then from (14) we get :

$$
\begin{aligned}
= & 4 \lim _{z \rightarrow z_{o}} \frac{d^{2}}{d z^{2}}\left[\frac{U(z) p^{(1)}(z)-p(z) U^{(1)}(z)}{(U(z))^{2}}\right] \\
= & 4 \lim _{z \rightarrow z_{o}} \frac{d}{d z}\left[\frac{U(z) p^{(2)}(z)-p^{(1)}(z) U^{(1)}(z)}{(U(z))^{2}}\right. \\
& \left.-\frac{p(z) U^{(2)}(z)+U^{(1)}(z) p^{(1)}(z)}{(U(z))^{2}}-2 \times \frac{\left(U^{(1)}(z)\right)^{2} p(z)}{(U(z))^{3}}\right] \\
= & 4 \lim _{z \rightarrow z_{o}}\left[\frac{p^{(3)}(z)}{U(z)}-3 \times \frac{p^{(2)}(z) U^{(1)}(z)}{(U(z))^{2}}\right. \\
& -\frac{p(z) U^{(3)}(z)}{(U(z))^{2}-3 \times \frac{p^{(1)}(z) U^{(2)}(z)}{(U(z))^{2}}+} \\
& 6 \times \frac{p(z) U^{(1)}(z) U^{(2)}(z)}{(U(z))^{3}}+6 \times \frac{\left(U^{(1)}(z)\right)^{2} p^{(1)}(z)}{(U(z))^{3}} \\
& \left.-6 \times \frac{\left(U^{(1)}(z)\right)^{3} p(z)}{(U(z))^{4}}\right]
\end{aligned}
$$

\section{Where}

$$
\begin{aligned}
& U(z)=q^{(4)}\left(z_{o}\right)+\frac{1}{5}\left(z-z_{o}\right) q^{(5)}\left(z_{o}\right)+\frac{1}{6 \times 5}\left(z-z_{o}\right)^{2} q^{(6)}\left(z_{o}\right)+\ldots \quad ; \lim _{z \rightarrow z_{o}} U(z)=q^{(4)}\left(z_{o}\right) \\
& U^{(1)}(z)=\frac{1}{5} q^{(5)}\left(z_{o}\right)+\frac{2}{6 \times 5}\left(z-z_{o}\right) q^{(6)}\left(z_{o}\right)+\ldots \ldots \ldots \ldots \ldots \ldots \ldots \ldots \ldots \\
& U^{(2)}(z)=\frac{2}{6 \times 5} q^{(6)}\left(z_{o}\right)+\frac{6}{7 \times 6 \times 5}\left(z-z_{o}\right) q^{(7)}\left(z_{o}\right)+\ldots \ldots . \quad ; \quad \lim _{z \rightarrow z_{o}} U^{(2)}(z)=\frac{1}{15} q^{(6)}\left(z_{o}\right) \\
& U^{(3)}(z)=\frac{6}{7 \times 6 \times 5} q^{(7)}\left(z_{o}\right)+\frac{24}{8 \times 7 \times 6 \times 5}\left(z-z_{o}\right) q^{(8)}\left(z_{o}\right)+\ldots . . ; \lim _{z \rightarrow z_{o}} U^{(3)}(z)=\frac{1}{35} q^{(7)}\left(z_{o}\right)
\end{aligned}
$$


Then substituting the above values in (19) in equation (18) we get the desired proof.

\section{3) In this approach we can find the residue for $(m=5)$ :}

If $\left(z=z_{o}\right)$ is a pole of order $(\mathrm{m}=5)$ i.e

$\left\lfloor q\left(z_{o}\right)=0, q^{\prime}\left(z_{o}\right)=0, q^{\prime \prime}\left(z_{o}\right)=0, q^{\prime \prime \prime}\left(z_{o}\right)=0, q^{\prime \prime \prime \prime}\left(z_{o}\right)=0, q^{\prime \prime \prime I I}\left(z_{o}\right) \neq 0\right\rfloor$ then the

residue is given by the following form :

$$
\begin{aligned}
\operatorname{Re} s\left(f, z_{o}\right) & =5 \times\left[\frac{p^{(4)}\left(z_{o}\right)}{q^{(5)}\left(z_{o}\right)}-4 \times \frac{\frac{1}{6} q^{(6)}\left(z_{o}\right) p^{(3)}\left(z_{o}\right)}{\left(q^{(5)}\left(z_{o}\right)\right)^{2}}\right. \\
& -6 \times \frac{\frac{1}{21} q^{(7)}\left(z_{o}\right) p^{(2)}(z)}{\left(q^{(5)}\left(z_{o}\right)\right)^{2}}+12 \times \frac{\left(\frac{1}{6} q^{(6)}\left(z_{o}\right)\right)^{2} p^{2}\left(z_{o}\right)}{\left(q^{(5)}\left(z_{o}\right)\right)^{3}} \\
& -\frac{\frac{1}{126} q^{(9)}\left(z_{o}\right) p\left(z_{o}\right)}{\left(q^{(5)}\left(z_{o}\right)\right)^{2}}-4 \times \frac{\frac{1}{56} q^{(8)}\left(z_{o}\right) p^{(1)}\left(z_{o}\right)}{\left(q^{(5)}\left(z_{o}\right)\right)^{2}} \\
& +\frac{\frac{1}{42} q^{(6)}\left(z_{o}\right) q^{(8)}\left(z_{o}\right) p\left(z_{o}\right)}{\left(q^{(5)}\left(z_{o}\right)\right)^{3}} \\
& +4 \times \frac{\frac{1}{21} q^{(6)}\left(z_{o}\right) q^{(7)}\left(z_{o}\right) p^{(1)}\left(z_{o}\right)}{\left(q^{(5)}\left(z_{o}\right)\right)^{3}}+6 \times \frac{\left(\frac{1}{21} q^{(7)}\left(z_{o}\right)\right)^{2} p\left(z_{o}\right)}{\left(q^{(5)}\left(z_{o}\right)\right)^{3}} \\
& -36 \times \frac{\left(\frac{1}{6} q^{(6)}\left(z_{o}\right)\right)^{2} \frac{1}{21} q^{(7)}\left(z_{o}\right) p\left(z_{o}\right)}{\left(q^{(5)}\left(z_{o}\right)\right)^{4}} \\
& -24 \times \frac{\left(\frac{1}{6} q^{(6)}\left(z_{o}\right)\right)^{3} p^{(1)}\left(z_{o}\right)}{\left(q^{(5)}\left(z_{o}\right)\right)^{4}}+24 \times \frac{\left(\frac{1}{6} q^{(6)}\left(z_{o}\right)\right)^{4} p\left(z_{o}\right)}{\left(q^{(5)}\left(z_{o}\right)\right)^{5}}
\end{aligned}
$$

Proof : (By the same way in $(\mathbf{m}=\mathbf{3}) \&(\mathbf{m}=4))$.

\section{4) In this approach we can find the residue for $(m=6)$ :}

If $\left(z=z_{o}\right)$ is a pole of order $(\mathrm{m}=6)$ i.e

$\left\lfloor q\left(z_{o}\right)=q^{(1)}\left(z_{o}\right)=q^{(2)}\left(z_{o}\right)=q^{(3)}\left(z_{o}\right)=q^{(4)}\left(z_{o}\right)=q^{(5)}\left(z_{o}\right)=0, q^{(6)}\left(z_{o}\right) \neq 0\right\rfloor$ then the residue is given by the following form : 


$$
\begin{aligned}
& \operatorname{Re} s\left(f, z_{o}\right)=6 \times\left[\frac{p^{(5)}\left(z_{o}\right)}{q^{(6)}\left(z_{o}\right)}-5 \times \frac{\frac{1}{7} p^{(4)}\left(z_{o}\right) q^{(7)}\left(z_{o}\right)}{\left(q^{(6)}\left(z_{o}\right)\right)^{2}}\right. \\
& -4 \times \frac{\frac{1}{28} p^{(3)}\left(z_{o}\right) q^{(8)}\left(z_{o}\right)}{\left(q^{(6)}\left(z_{o}\right)\right)^{2}}-10 \times \frac{\frac{1}{84} p^{(2)}\left(z_{o}\right) q^{(9)}\left(z_{o}\right)}{\left(q^{(6)}\left(z_{o}\right)\right)^{2}} \\
& -6 \times \frac{\frac{1}{28} p^{(2)}\left(z_{o}\right) q^{(8)}\left(z_{o}\right)}{\left(q^{(6)}\left(z_{o}\right)\right)^{2}}-5 \times \frac{\frac{1}{210} p^{(1)}\left(z_{o}\right) q^{(10)}\left(z_{o}\right)}{\left(q^{(6)}\left(z_{o}\right)\right)^{2}} \\
& -\frac{\frac{1}{462} p\left(z_{o}\right) q^{(11)}\left(z_{o}\right)}{\left(q^{(6)}\left(z_{o}\right)\right)^{2}}+20 \times \frac{\left(\frac{1}{7} q^{(7)}\left(z_{o}\right)\right)^{2} p^{(3)}\left(z_{o}\right)}{\left(q^{(6)}\left(z_{o}\right)\right)^{3}} \\
& +60 \times \frac{\frac{1}{196} q^{(7)}\left(z_{o}\right) q^{(8)}\left(z_{o}\right) p^{(2)}\left(z_{o}\right)}{\left(q^{(6)}\left(z_{o}\right)\right)^{3}}+10 \times \frac{\left(\frac{1}{7} \times \frac{1}{210}\right) p\left(z_{o}\right) q^{(7)}\left(z_{o}\right) q^{(10)}\left(z_{o}\right)}{\left(q^{(6)}\left(z_{o}\right)\right)^{3}} \\
& +8 \times \frac{\frac{1}{196} q^{(7)}\left(z_{o}\right) q^{(8)}\left(z_{o}\right) p^{(1)}\left(z_{o}\right)}{\left(q^{(6)}\left(z_{o}\right)\right)^{3}}+20 \times \frac{\left(\frac{1}{84} \times \frac{1}{28}\right) q^{(9)}\left(z_{o}\right) q^{(8)}\left(z_{o}\right) p\left(z_{o}\right)}{\left(q^{(6)}\left(z_{o}\right)\right)^{3}} \\
& +32 \frac{\left(\frac{1}{196}\right) q^{(7)}\left(z_{o}\right) q^{(9)}\left(z_{o}\right) p^{(1)}\left(z_{o}\right)}{\left(q^{(6)}\left(z_{o}\right)\right)^{3}}+24 \times \frac{\frac{1}{196} p^{(1)}\left(z_{o}\right) q^{(7)}\left(z_{o}\right) q^{(9)}\left(z_{o}\right)}{\left(q^{(6)}\left(z_{o}\right)\right)^{3}} \\
& +30 \times \frac{\left(\frac{1}{28} q^{(8)}\left(z_{o}\right)\right)^{2} p^{(1)}\left(z_{o}\right)}{\left(q^{(6)}\left(z_{o}\right)\right)^{3}}-60 \times \frac{\left(\frac{1}{7} q^{(7)}\left(z_{o}\right)\right)^{3} p^{(2)}\left(z_{o}\right)}{\left(q^{(6)}\left(z_{o}\right)\right)^{4}} \\
& -60 \times \frac{\left(\frac{1}{7} q^{(7)}\left(z_{o}\right)\right)^{2} \frac{1}{84} q^{(9)}\left(z_{o}\right) p\left(z_{o}\right)}{\left(q^{(6)}\left(z_{o}\right)\right)^{4}}-180 \times \frac{\left(\frac{1}{7} q^{(7)}\left(z_{o}\right)\right)^{2} \frac{1}{28} q^{(8)}\left(z_{o}\right) p^{(1)}\left(z_{o}\right)}{\left(q^{(6)}\left(z_{o}\right)\right)^{4}} \\
& -90 \times \frac{\frac{1}{7} q^{(7)}\left(z_{o}\right)\left(\frac{1}{28} q^{(8)}\left(z_{o}\right)\right)^{2} p\left(z_{o}\right)}{\left(q^{(6)}\left(z_{o}\right)\right)^{4}}+240 \times \frac{\left(\frac{1}{7} q^{(7)}\left(z_{o}\right)\right)^{3} \frac{1}{28} q^{(8)}\left(z_{o}\right) p\left(z_{0}\right)}{\left(q^{(6)}\left(z_{o}\right)\right)^{5}} \\
& \left.+120 \times \frac{\left(\frac{1}{7} q^{(7)}\left(z_{o}\right)\right)^{4} p^{(1)}\left(z_{o}\right)}{\left(q^{(6)}\left(z_{o}\right)\right)^{5}}-120 \times \frac{\left(\frac{1}{7} q^{(7)}\left(z_{o}\right)\right)^{5} p\left(z_{o}\right)}{\left(q^{(6)}\left(z_{o}\right)\right)^{6}}\right]
\end{aligned}
$$

Proof: (By the same way in $(\mathbf{m}=3) \&(\mathbf{m}=4))$.

3.5) If $\left(z=z_{o}\right)$ is a pole of order $(\mathrm{m}=7)$ i.e $\left\lfloor q\left(z_{o}\right)=q^{(1)}\left(z_{o}\right)=q^{(2)}\left(z_{o}\right)=q^{(3)}\left(z_{o}\right)=q^{(4)}(z)=q^{(5)}\left(z_{o}\right)=q^{(6)}\left(z_{o}\right)=0, q^{(7)}\left(z_{o}\right) \neq 0\right\rfloor$ then the residue is given by the following form: 


$$
\begin{aligned}
& \operatorname{Re} s(f, z)=7 \times\left[\frac{p^{(6)}\left(z_{o}\right)}{q^{(7)}\left(z_{o}\right)}-6 \times \frac{p^{(5)}\left(z_{o}\right) \frac{1}{8} q^{(8)}\left(z_{o}\right)}{\left(q^{(7)}\left(z_{o}\right)\right)^{2}}\right. \\
& -9 \times \frac{p^{(4)}\left(z_{o}\right) \frac{1}{36} q^{(9)}\left(z_{o}\right)}{\left(q^{(7)}\left(z_{o}\right)\right)^{2}}-14 \times \frac{p^{(3)}\left(z_{o}\right) \frac{1}{120} q^{(10)}\left(z_{o}\right)}{\left(q^{(7)}\left(z_{o}\right)\right)^{2}}- \\
& -15 \times \frac{\frac{1}{330} q^{(11)}\left(z_{o}\right) p^{(2)}\left(z_{o}\right)}{\left(q^{(7)}\left(z_{o}\right)\right)^{2}}-6 \times \frac{p^{(2)}\left(z_{o}\right) \frac{1}{120} q^{(10)}\left(z_{o}\right)}{\left(q^{(7)}\left(z_{o}\right)\right)^{2}} \\
& -6 \times \frac{p^{(3)}\left(z_{o}\right) \frac{1}{36} q^{(9)}\left(z_{o}\right)}{\left(q^{(7)}\left(z_{o}\right)\right)^{2}}-6 \times \frac{p^{(1)}\left(z_{o}\right) \frac{1}{792} q^{(12)}\left(z_{o}\right)}{\left(q^{(7)}\left(z_{o}\right)\right)^{2}}- \\
& \frac{p\left(z_{o}\right) \frac{1}{1716} q^{(13)}\left(z_{o}\right)}{\left(q^{(7)}\left(z_{o}\right)\right)^{2}}+20 \times \frac{\left(\frac{1}{8} \times \frac{1}{36}\right) q^{(8)}\left(z_{o}\right) q^{(9)}\left(z_{o}\right) p^{(2)}\left(z_{o}\right)}{\left(q^{(7)}\left(z_{o}\right)\right)^{3}} \\
& +30 \times \frac{\left(\frac{1}{8} q^{(8)}\left(z_{o}\right)\right)^{2} p^{(4)}\left(z_{o}\right)}{\left(q^{(7)}\left(z_{o}\right)\right)^{3}}+108 \times \frac{\left(\frac{1}{8} \times \frac{1}{36}\right) q^{(8)}\left(z_{o}\right) q^{(9)}\left(z_{o}\right) p^{(3)}\left(z_{o}\right)}{\left(q^{(7)}\left(z_{o}\right)\right)^{3}} \\
& +80 \times \frac{\left(\frac{1}{8} \times \frac{1}{120}\right) q^{(8)}\left(z_{o}\right) q^{(10)}\left(z_{o}\right) p^{(2)}\left(z_{0}\right)}{\left(q^{(7)}\left(z_{o}\right)\right)^{3}}+76 \times \frac{\left(\frac{1}{8} \times \frac{1}{330}\right) q^{(8)}\left(z_{o}\right) q^{(11)}\left(z_{o}\right) p^{(1)}\left(z_{o}\right)}{\left(q^{(7)}\left(z_{o}\right)\right)^{3}} \\
& +12 \times \frac{\left(\frac{1}{8} \times \frac{1}{792}\right) q^{(8)}\left(z_{o}\right) q^{(12)}\left(z_{o}\right) p\left(z_{o}\right)}{\left(q^{(7)}\left(z_{o}\right)\right)^{3}}+90 \times \frac{\left(\frac{1}{36} q^{(9)}\left(z_{o}\right)\right)^{2} p^{(2)}\left(z_{o}\right)}{\left(q^{(7)}\left(z_{o}\right)\right)^{3}}+ \\
& +30 \times \frac{\left(\frac{1}{36} \times \frac{1}{330}\right) p\left(z_{o}\right) q^{(11)}\left(z_{o}\right) q^{(9)}\left(z_{o}\right)}{\left(q^{(7)}\left(z_{o}\right)\right)^{3}}+8 \times \frac{\left(\frac{1}{8} \times \frac{1}{120}\right) q^{(8)}\left(z_{o}\right) q^{(10)}\left(z_{o}\right) p^{(1)}\left(z_{o}\right)}{\left(q^{(7)}\left(z_{o}\right)\right)^{3}} \\
& +8 \times \frac{\left(\frac{1}{36} q^{(9)}\left(z_{o}\right)\right)^{2} p^{(1)}\left(z_{o}\right)}{\left(q^{(7)}\left(z_{o}\right)\right)^{3}}+136 \times \frac{\left(\frac{1}{36} \times \frac{1}{120}\right) q^{(9)}\left(z_{o}\right) q^{(10)}\left(z_{o}\right) p^{(1)}\left(z_{o}\right)}{\left(q^{(7)}\left(z_{o}\right)\right)^{3}} \\
& +20 \times \frac{\left(\frac{1}{120} q^{(10)}\left(z_{o}\right)\right)^{2} p\left(z_{o}\right)}{\left(q^{(7)}\left(z_{o}\right)\right)^{3}}+56 \frac{\left(\frac{1}{8} \times \frac{1}{120}\right) q^{(8)}\left(z_{o}\right) q^{(10)}\left(z_{o}\right) p^{(2)}\left(z_{o}\right)}{\left(q^{(7)}\left(z_{o}\right)\right)^{3}}
\end{aligned}
$$




$$
\begin{aligned}
& -120 \times \frac{\left(\frac{1}{8} q^{(8)}\left(z_{o}\right)\right)^{3} p^{(3)}\left(z_{o}\right)}{\left(q^{(7)}\left(z_{o}\right)\right)^{4}}-540 \times \frac{\left(\frac{1}{8} q^{(8)}\left(z_{o}\right)\right)^{2} \frac{1}{36} q^{(9)}\left(z_{o}\right) p^{(2)}\left(z_{o}\right)}{\left(q^{(7)}\left(z_{o}\right)\right)^{4}} \\
& -90 \times \frac{\left(\frac{1}{8} q^{(8)}\left(z_{o}\right)\right)^{2} \frac{1}{330} q^{(11)}\left(z_{o}\right) p\left(z_{o}\right)}{\left(q^{(7)}\left(z_{o}\right)\right)^{4}}-24 \times \frac{\left(\frac{1}{8} q^{(8)}\left(z_{o}\right)\right)^{2} \frac{1}{36} q^{(9)}\left(z_{o}\right) p^{(1)}\left(z_{o}\right)}{\left(q^{(7)}\left(z_{o}\right)\right)^{4}} \\
& -360 \times \frac{\left(\frac{1}{8} \times \frac{1}{36} \times \frac{1}{120}\right) q^{(8)}\left(z_{o}\right) q^{(9)}\left(z_{o}\right) q^{(10)}\left(z_{o}\right) p\left(z_{o}\right)}{\left(q^{(7)}\left(z_{o}\right)\right)^{4}}- \\
& -540 \times \frac{\left(\frac{1}{36} q^{(9)}\left(z_{o}\right)\right)^{2} \frac{1}{8} q^{(8)}\left(z_{o}\right) p^{(1)}\left(z_{o}\right)}{\left(q^{(7)}\left(z_{o}\right)\right)^{4}}-90 \times \frac{\left(\frac{1}{36} q^{(9)}\left(z_{o}\right)\right)^{2} p\left(z_{o}\right)}{\left(q^{(7)}\left(z_{o}\right)\right)^{4}} \\
& +360 \times \frac{\left(\frac{1}{8} q^{(8)}\left(z_{o}\right)\right)^{4} p^{(2)}\left(z_{o}\right)}{\left(q^{(7)}\left(z_{o}\right)\right)^{5}}+480 \times \frac{\left(\frac{1}{8} q^{(8)}\left(z_{o}\right)\right)^{3} \frac{1}{120} q^{(10)}\left(z_{o}\right) p\left(z_{o}\right)}{\left(q^{(7)}\left(z_{o}\right)\right)^{5}} \\
& +1440 \times \frac{\left(\frac{1}{8} q^{(8)}\left(z_{o}\right)\right)^{3} \frac{1}{36} q^{(9)}\left(z_{o}\right) p^{(1)}\left(z_{o}\right)}{\left(q^{(7)}\left(z_{o}\right)\right)^{5}}+ \\
& 1080 \times \frac{\left(\frac{1}{36} q^{(9)}\left(z_{o}\right)\right)^{2}\left(\frac{1}{8} q^{(8)}\left(z_{o}\right)\right)^{2} p\left(z_{o}\right)}{\left(q^{(7)}\left(z_{o}\right)\right)^{5}}-1800 \times \frac{\left(\frac{1}{8} q^{(8)}\left(z_{o}\right)\right)^{4} \frac{1}{36} q^{(9)}\left(z_{o}\right) p\left(z_{o}\right)}{\left(q^{(7)}\left(z_{o}\right)\right)^{6}}- \\
& \left.720 \times \frac{\left(\frac{1}{8} q^{(8)}\left(z_{o}\right)\right)^{5} p^{(1)}\left(z_{o}\right)}{\left(q^{(7)}\left(z_{o}\right)\right)^{6}}+720 \times \frac{\left(\frac{1}{8} q^{(8)}\left(z_{o}\right)\right)^{6} p\left(z_{o}\right)}{\left(q^{(7)}\left(z_{o}\right)\right)^{7}}\right]
\end{aligned}
$$

\section{Proof:}

If $\left(z=z_{o}\right)$ is a pole of order $(\mathbf{m}=7)$ then by (short - cut -method) we get :

$\operatorname{Re} s\left(f, z_{o}\right)=\frac{1}{6 !} \lim _{z \rightarrow z_{o}} \frac{d^{6}}{d z^{6}}\left[\left(z-z_{o}\right)^{7} \frac{p(z)}{q(z)}\right]$ where $\quad\left(f(z)=\frac{p(z)}{q(z)}\right)$

We expand the analytic function $\mathrm{q}(\mathrm{z})$ into Taylor series valid in disk $\left|z-z_{o}\right|<r$

$$
=\frac{1}{6 !} \lim _{z \rightarrow z_{o}} \frac{d^{6}}{d z^{6}}\left[\frac{\left(z-z_{o}\right)^{7} p(z)}{q\left(z_{o}\right)+\left(z-z_{o}\right) q^{(1)}\left(z_{o}\right)+\frac{\left(z-z_{o}\right)^{2}}{2 !} q^{(2)}\left(z_{0}\right)+\frac{\left(z-z_{o}\right)^{3}}{3 !} q^{(3)}\left(z_{o}\right)+\ldots \ldots .}\right] \ldots
$$




$$
=7 \lim _{z \rightarrow z_{o}} \frac{d^{6}}{d z^{6}}\left[\frac{p(z)}{U(z)}\right]
$$

where :

$U(z)=q^{(7)}\left(z_{o}\right)+\frac{\left(z-z_{o}\right)}{8} q^{(8)}\left(z_{o}\right)+\frac{\left(z-z_{o}\right)^{2}}{8 \times 9} q^{(9)}\left(z_{o}\right)+\frac{\left(z-z_{o}\right)^{3}}{8 \times 9 \times 10} q^{(10)}\left(z_{o}\right)+\ldots \ldots$

Then from (25) we get :

$=7 \lim _{z \rightarrow z_{o}} \frac{d^{5}}{d z^{5}}\left[\frac{U(z) p^{\prime}(z)-p(z) U^{(1)}(z)}{(U(z))^{2}}\right]$

$=7 \lim _{z \rightarrow z_{o}} \frac{d^{4}}{d z^{4}}\left[\frac{p^{(2)}(z)}{U(z)}-\frac{p(z) U^{(2)}(z)}{(U(z))^{2}}-2 \times \frac{p^{(1)}(z) U^{(1)}(z)}{(U(z))^{2}}+2 \times \frac{p(z)\left(U^{(1)}(z)\right)^{2}}{(U(z))^{3}}\right]$

$=7 \lim _{z \rightarrow z_{o}} \frac{d^{3}}{d z^{3}}\left[\frac{p^{(3)}(z)}{U(z)}-3 \times \frac{p^{(2)}(z) U^{(1)}(z)}{(U(z))^{2}}-\frac{p(z) U^{(3)}(z)}{(U(z))^{2}}-3 \times \frac{p(z) U^{(2)}(z)}{(U(z))^{2}}\right.$

$\left.+6 \times \frac{p(z) U^{(1)}(z) U^{(2)}(z)}{(U(z))^{3}}+6 \times \frac{p^{(1)}(z)\left(U^{(1)}(z)\right)^{2}}{(U(z))^{3}}-6 \times \frac{p(z)\left(U^{(1)}(z)\right)^{3}}{(U(z))^{4}}\right]$

$=7 \lim _{z \rightarrow z_{o}} \frac{d^{2}}{d z^{2}}\left[\frac{p^{(4)}(z)}{U(z)}-4 \times \frac{p^{(3)}(z) U^{(1)}(z)}{(U(z))^{2}}\right.$

$-6 \times \frac{p^{(2)}(z) U^{(2)}(z)}{(U(z))^{2}}+12 \times \frac{p^{(2)}(z)\left(U^{(1)}(z)\right)^{2}}{(U(z))^{3}}$

$-\frac{p(z) U^{(4)}(z)}{(U(z))^{2}}-4 \times \frac{p^{(1)}(z) U^{(3)}(z)}{(U(z))^{2}}+8 \times \frac{p(z) U^{(1)}(z) U^{(3)}(z)}{(U(z))^{3}}$

$+24 \times \frac{p^{(1)}(z) U^{(1)}(z) U^{(2)}(z)}{(U(z))^{3}}+$

$$
\begin{aligned}
& 6 \times \frac{p(z)\left(U^{(2)}(z)\right)^{2}}{(U(z))^{3}}-36 \times \frac{p(z)\left(U^{(1)}(z)\right)^{2} U^{(2)}(z)}{(U(z))^{4}} \\
& \left.-24 \times \frac{p^{(1)}(z)\left(U^{(1)}(z)\right)^{3}}{(U(z))^{4}}+24 \frac{p(z)\left(U^{(1)}(z)\right)^{4}}{(U(z))^{5}}\right] \\
& =7 \lim _{z \rightarrow z_{o}} \frac{d}{d z}\left[\frac{p^{(5)}(z)}{U(z)}-5 \times \frac{p^{(4)}(z) U^{(1)}(z)}{(U(z))^{2}}-4 \times \frac{p^{(3)}(z) U^{(2)}(z)}{(U(z))^{2}}-10 \times \frac{p^{(2)}(z) U^{(3)}(z)}{(U(z))^{2}}-\right.
\end{aligned}
$$




$$
\begin{aligned}
& 6 \times \frac{p^{(2)}(z) U^{(2)}(z)}{(U(z))^{2}} 5 \times \frac{p^{(1)}(z) U^{(4)}(z)}{(U(z))^{2}}-\frac{p(z) U^{(5)}(z)}{(U(z))^{2}}+20 \times \frac{\left(U^{(1)}(z)\right)^{2} p^{(3)}(z)}{(U(z))^{3}} \\
& +60 \times \frac{U^{(1)}(z) U^{(2)}(z) p^{(2)}(z)}{(U(z))^{3}}+10 \times \frac{p(z) U^{(1)}(z) U^{(4)}(z)}{(U(z))^{3}}+8 \times \frac{U^{(1)}(z) U^{(2)}(z) p^{(1)}(z)}{(U(z))^{3}} \\
& +20 \times \frac{U^{(3)}(z) U^{(2)}(z) p(z)}{(U(z))^{3}}+32 \times \frac{U^{(1)}(z) U^{(3)}(z) p^{(1)}(z)}{(U(z))^{3}}+24 \times \frac{p^{(1)}(z) U^{(1)}(z) U^{(3)}(z)}{(U(z))^{3}} \\
& +30 \times \frac{p^{(1)}(z)\left(U^{(2)}(z)\right)^{2}}{(U(z))^{3}}-60 \times \frac{\left(U^{(1)}(z)\right)^{3} p^{(2)}(z)}{(U(z))^{4}}-60 \times \frac{\left(U^{(1)}(z)\right)^{2} U^{(3)}(z) p(z)}{(U(z))^{4}} \\
& -180 \times \frac{\left(U^{(1)}(z)\right)^{2} U^{(2)}(z) p^{(1)}(z)}{(U(z))^{4}}-90 \times \frac{U^{(1)}(z)\left(U^{(2)}(z)\right)^{2} p(z)}{(U(z))^{4}}+240 \times \frac{\left(U^{(1)}(z)\right)^{3} U^{(2)}(z) p(z)}{(U(z))^{5}} \\
& +120 \times \frac{\left(U^{(1)}(z)\right)^{4} p^{(1)}(z)}{(U(z))^{5}}-120 \times \frac{\left(U^{(1)}(z)\right)^{5} p(z)}{(U(z))^{6}} \\
& =7 \lim _{z \rightarrow z_{o}}\left[\frac{p^{(6)}(z)}{U(z)}-6 \times \frac{p^{(5)}(z) U^{(1)}(z)}{(U(z))^{2}}-9 \times \frac{p^{(4)}(z) U^{(2)}(z)}{(U(z))^{2}}-14 \times \frac{p^{(3)}(z) U^{(3)}(z)}{(U(z))^{2}}-\right. \\
& 15 \times \frac{U^{(4)}(z) p^{(2)}(z)}{(U(z))^{2}}-6 \times \frac{p^{(2)}(z) U^{(3)}(z)}{(U(z))^{2}}-6 \times \frac{p^{(3)}(z) U^{(2)}(z)}{(U(z))^{2}}+20 \times \frac{U^{(1)}(z) U^{(2)}(z) p^{(2)}(z)}{(U(z))^{3}} \\
& -6 \times \frac{p^{(1)}(z) U^{(5)}(z)}{(U(z))^{2}}-\frac{p(z) U^{(6)}(z)}{(U(z))^{2}}+30 \times \frac{\left(U^{(1)}(z)\right)^{2} p^{(4)}(z)}{(U(z))^{3}}+108 \times \frac{U^{(1)}(z) U^{(2)}(z) p^{(3)}(z)}{(U(z))^{3}} \\
& +80 \times \frac{U^{(1)}(z) U^{(3)}(z) p^{(2)}(z)}{(U(z))^{3}}+76 \times \frac{U^{(1)}(z) U^{(4)}(z) p^{(1)}(z)}{(U(z))^{3}}+12 \times \frac{U^{(1)}(z) U^{(5)}(z) p(z)}{(U(z))^{3}} \\
& +90 \times \frac{p^{(2)}(z)\left(U^{(2)}(z)\right)^{2}}{(U(z))^{3}}+30 \times \frac{p(z) U^{(2)}(z) U^{(4)}(z)}{(U(z))^{3}}+8 \times \frac{U^{(1)}(z) U^{(3)}(z) p^{(1)}(z)}{(U(z))^{3}} \\
& +8 \times \frac{p^{(1)}(z)\left(U^{(2)}(z)\right)^{2}}{(U(z))^{3}}
\end{aligned}
$$




$$
\begin{aligned}
& +136 \times \frac{U^{(2)}(z) U^{(3)}(z) p^{(1)}(z)}{(U(z))^{3}}+20 \times \frac{\left(U^{(3)}(z)\right)^{2} p(z)}{(U(z))^{3}} \\
& +56 \times \frac{U^{(1)}(z) U^{(3)}(z) p^{(2)}(z)}{(U(z))^{3}}-120 \times \frac{\left(U^{(1)}(z)\right)^{3} p^{(3)}(z)}{(U(z))^{4}} \\
& -540 \times \frac{\left(U^{(1)}(z)\right)^{2} U^{(2)}(z) p^{(2)}(z)}{(U(z))^{4}}-90 \times \frac{\left(U^{(1)}(z)\right)^{2} U^{(4)}(z) p(z)}{(U(z))^{4}} \\
& -24 \times \frac{\left(U^{(1)}(z)\right)^{2} U^{(2)}(z) p^{(1)}(z)}{(U(z))^{4}}- \\
& 360 \times \frac{U^{(1)}(z) U^{(2)}(z) U^{(3)}(z) p(z)}{(U(z))^{4}}+408 \times \frac{\left(U^{(1)}(z)\right)^{2} U^{(3)}(z) p^{(1)}(z)}{(U(z))^{4}} \\
& -540 \times \frac{U^{(1)}(z)\left(U^{(2)}(z)\right)^{2} p^{(1)}(z)}{(U(z))^{4}}-90 \times \frac{\left(U^{(2)}(z)\right)^{2} p(z)}{(U(z))^{4}} \\
& +360 \times \frac{\left(U^{(1)}(z)\right)^{4} p^{(2)}(z)}{(U(z))^{5}}+480 \times \frac{\left(U^{(1)}(z)\right)^{4} U^{(3)}(z) p(z)}{(U(z))^{5}}+ \\
& 1440 \times \frac{\left(U^{(1)}(z)\right)^{3} U^{(2)}(z) p^{(1)}(z)}{(U(z))^{5}}+1080 \times \frac{\left(U^{(2)}(z)\right)^{2}\left(U^{(1)}(z)\right)^{2} p(z)}{(U(z))^{5}} \\
& \left.-1800 \times \frac{\left(U^{(1)}(z)\right)^{4} U^{(2)}(z) p(z)}{(U(z))^{6}}-720 \times \frac{\left(U^{(1)}(z)\right)^{5} p^{(1)}(z)}{(U(z))^{6}}+720 \times \frac{\left(U^{(1)}(z)\right)^{6} p(z)}{(U(z))^{7}}\right]
\end{aligned}
$$

Where : 


$$
\begin{aligned}
& U(z)=q^{(7)}\left(z_{o}\right)+\frac{\left(z-z_{o}\right)}{8} q^{(8)}\left(z_{o}\right)+\frac{\left(z-z_{o}\right)^{2}}{9 \times 8} q^{(9)}\left(z_{o}\right)+\ldots \\
& \lim _{z \rightarrow z_{o}} U(z)=q^{(7)}\left(z_{o}\right) \\
& U^{(1)}(z)=\frac{1}{8} q^{(8)}\left(z_{o}\right)+\frac{2}{9 \times 8}\left(z-z_{o}\right) q^{(9)}\left(z_{o}\right)+\ldots \ldots \ldots \\
& \lim _{z \rightarrow z_{o}} U^{(1)}(z)=\frac{1}{8} q^{(8)}\left(z_{o}\right) \\
& U^{(2)}(z)=\frac{2}{9 \times 8} q^{(9)}\left(z_{o}\right)+\frac{3 \times 2}{10 \times 9 \times 8}\left(z-z_{o}\right) q^{(10)}\left(z_{o}\right)+\ldots \\
& \lim _{z \rightarrow z_{o}} U^{(2)}(z)=\frac{1}{36} q^{(9)}\left(z_{o}\right) \\
& U^{(3)}(z)=\frac{3 \times 2}{10 \times 9 \times 8} q^{(10)}\left(z_{o}\right)+\frac{4 \times 3 \times 2}{11 \times 10 \times 9 \times 8}\left(z-z_{o}\right) q^{(11)}\left(z_{o}\right)+\ldots \\
& \lim _{z \rightarrow z_{o}} U^{(3)}(z)=\frac{1}{120} q^{(10)}\left(z_{o}\right) \\
& U^{(4)}(z)=\frac{4 \times 3 \times 2}{11 \times 10 \times 9 \times 8} q^{(11)}\left(z_{o}\right)+\frac{5 \times 4 \times 3 \times 2}{12 \times 11 \times 10 \times 9 \times 8}\left(z-z_{o}\right) q^{(12)}\left(z_{o}\right)+\ldots \ldots \\
& \lim _{z \rightarrow z_{o}} U^{(4)}(z)=\frac{1}{330} q^{(11)}\left(z_{o}\right) \\
& U^{(5)}(z)=\frac{5 \times 4 \times 3 \times 2}{12 \times 11 \times 10 \times 9 \times 8} q^{(12)}\left(z_{o}\right)+\frac{6 \times 5 \times 4 \times 3 \times 2}{13 \times 12 \times 11 \times 10 \times 9 \times 8}\left(z-z_{o}\right) q^{(13)}\left(z_{o}\right)+\ldots \\
& \lim _{z \rightarrow z_{o}} U^{(5)}(z)=\frac{1}{792} q^{(12)}\left(z_{o}\right) \\
& U^{(6)}(z)=\frac{6 \times 5 \times 4 \times 3 \times 2}{13 \times 12 \times 11 \times 10 \times 9 \times 8} q^{(13)}\left(z_{o}\right)+\frac{7 \times 6 \times 5 \times 4 \times 3 \times 2}{14 \times 13 \times 12 \times 11 \times 10 \times 9 \times 8}\left(z-z_{o}\right) q^{(14)}\left(z_{o}\right)+\ldots \\
& \lim _{z \rightarrow z_{o}} U^{(6)}(z)=\frac{1}{1716} q^{(13)}\left(z_{o}\right)
\end{aligned}
$$

Then substituting the above values in (33) in equation (32) we get the desired proof .

\section{6) In the above manner the procedure can be easily extended for any} pole of order (m) .

\section{4-Numarical computations :}

These examples can be found in $[1,4,6,7,8,10,11]$.

\section{Example (4.1) :}

Evaluate the following integral $\int_{C} \frac{Z^{2}+1}{(Z-2)^{4}(Z-1)^{3}} d Z$ at $\quad C: / Z /=3$. 
Solution:

The function $f(Z)$ has two poles $(Z=1, Z=2)$ in the region $c: / Z /=3$, and the order of these poles is $(m=3) \&(m=4)$, respectively

The solution by Cauchy integral formu
$\int_{C: I / Z=3} \frac{Z^{2}+1}{(Z-2)^{4}(Z-1)^{3}} d Z=58 \pi i-58 \pi i=0$

$\mathrm{cc}$

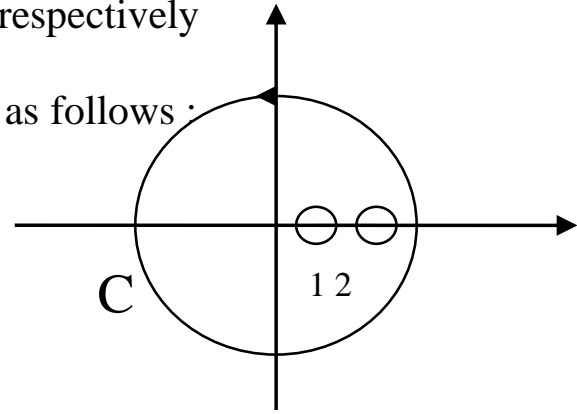

Figure 4.1

Then the solution by the new procedure is given as follows :

$\int_{C: / Z /=3} \frac{Z^{2}+1}{(Z-2)^{4}(Z-1)^{3}} d Z=2 \pi i \sum_{i=1}^{2} \operatorname{Re} s\left(f, Z_{i}\right)=2 \pi i \operatorname{Re} s\left(f, Z_{1}=1\right)+2 \pi i \operatorname{Re} s\left(f, Z_{2}=2\right)$

Where $p(Z)=Z^{2}+1 \quad ; \quad q(Z)=(Z-2)^{4}(Z-1)^{3}$

\begin{tabular}{|l|l|l|l|}
\hline $\mathrm{P}(2)=5$ & $q^{(4)}(2)=24$ & $\mathrm{P}(1)=2$ & $q^{(3)}(1)=6$ \\
\hline$p^{(1)}(2)=4$ & $q^{(5)}(2)=360$ & $p^{(1)}(1)=2$ & $q^{(4)}(1)=-96$ \\
\hline$p^{(2)}(2)=2$ & $q^{(6)}(2)=2160$ & $p^{(2)}(1)=2$ & $q^{(5)}(1)=720$ \\
\hline$p^{(3)}(2)=0$ & $q^{(7)}(2)=5040$ & $p^{(3)}(3)=0$ & \\
\hline
\end{tabular}

Table 4.1

Then by using the new procedure for $(m=3)$ we get :

$\operatorname{Re} s(f, 1)=3 \times\left[\frac{1}{3}-4+2.66+\frac{2304}{(36 \times 6)}\right]=28.98 \cong 29$

Then by using the new procedure for $(\mathrm{m}=4)$ we get

$\operatorname{Re} s(f, 2)=4 \times[-0.75-1.25-3+22.5+9-33.75]=-29$

Then :

$$
\begin{gathered}
\int_{C: / Z /=3} \frac{Z^{2}+1}{(Z-2)^{4}(Z-1)^{3}} d Z=2 \pi i \sum_{i=1}^{2} \operatorname{Re} s\left(f, Z_{i}\right)=2 \pi i \operatorname{Re} s\left(f, Z_{1}=1\right)+2 \pi i \operatorname{Re} s\left(f, Z_{2}=2\right) \\
=58 \pi i-58 \pi i \cong 0
\end{gathered}
$$

Example (4.2):

Evaluate the following integral $\int_{c} \frac{e^{Z}-Z^{6}}{(Z-2 i)^{6}} d z$ at $\mathrm{C}: / \mathrm{Z} /=3$.

\section{Solution:}

the function $f(z)$ has a pole $Z=2 i$ of order $(m=6)$. 
then by Cauchy integral formula we get:

$\int_{C: / Z /=3} \frac{e^{Z}-Z^{6}}{(Z-2 i)^{6}} d z=\frac{\pi i}{60}\left(e^{2 i}-1440 i\right)$.

Then by using the new procedure for $(\mathrm{m}=6)$ we get

$\int_{C: / Z /=3} \frac{e^{z}-Z^{6}}{(z-2 i)^{6}} d z=2 \pi i \operatorname{Re} s(f, 2 i)=\frac{\pi i}{60}\left(e^{2 i}-1440 i\right)$.

\section{Example (4.3)}

Evaluate the following improper integral $\int_{0}^{\infty} \frac{\sin (x)}{x^{m}} d x$ where $\mathrm{m}=1,3,5, \ldots \ldots$

\section{Solution:}

Now for this type of improper integrations we not can find the result by Cauchy integral formula. The only way to evaluate is the new procedure with general order $(\mathrm{m})$.

If $(\mathbf{m}=\mathbf{1})$ then $\int_{0}^{\infty} \frac{\sin (x)}{x} d x=\frac{\pi}{2}$

If $(\mathbf{m}=\mathbf{3})$ then $\int_{0}^{\infty} \frac{\sin (x)}{x^{3}} d x=\frac{-\pi}{4}$

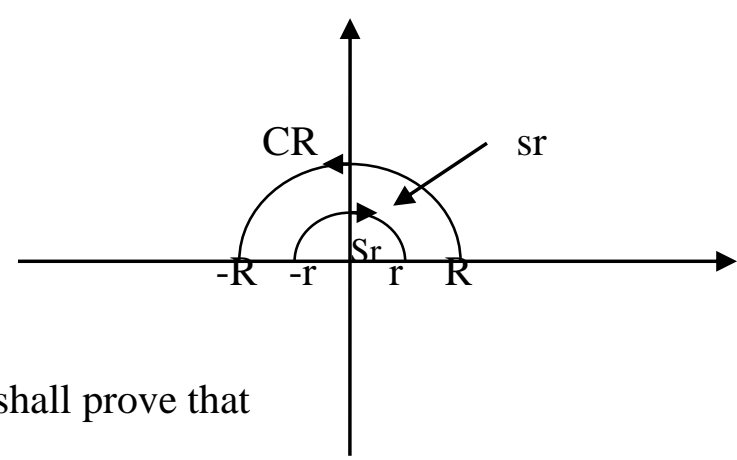

If $(\mathbf{m}=\mathbf{5})$ then $\int_{0}^{\infty} \frac{\sin (x)}{x^{5}}=\frac{\pi}{48}$ we shall prove that

\section{Figure 4.2}

The function $\mathrm{f}(\mathrm{Z})=\frac{e^{Z}}{Z^{5}}$ has pole $(\mathrm{Z}=0)$ of order $(\mathrm{m}=5)$, on real line . Then $\int_{0}^{\infty} \frac{\sin (x)}{x^{5}} d x=\lim _{R \rightarrow \infty} \int_{0}^{R} \frac{\sin (x)}{x^{5}} d x$ then by Jordan lemma we get:

$\lim _{R \rightarrow \infty, r \rightarrow 0}\left[\left(\int_{-R}^{-r}+\int_{r}^{R}+\int_{S r}+\int_{C R}\right) \frac{\sin (x)}{x^{5}} d x\right]=0$

Where

$\int \frac{\sin (x)}{x^{5}} d x=\int \frac{e^{i Z}}{Z^{5}} d Z$ on real part (x-axis). Then by lemma we get $\left(\int_{C R} \frac{e^{i Z}}{Z^{5}} d z=0\right)$ then

By replacing $(x=-x)$ in the above first term in the LHS then we have : 
$\lim _{R \rightarrow \infty, r \rightarrow 0}\left[2 i \int_{r}^{R} \frac{\sin (x)}{x^{5}} d x\right]=-\int_{S r} \frac{e^{i Z}}{Z^{5}} d z=\pi i \operatorname{Re} s(f, 0)$ then we find the residue at a

pole $(\mathrm{z}=0)$ of order $(\mathrm{m}=5)$.

Using the new procedure to find the residue for order $(\mathrm{m}=5)$

Now $\quad \mathrm{p}(\mathrm{z})=e^{i Z} \quad \& \quad \mathrm{q}(\mathrm{z})=Z^{5}$

\begin{tabular}{|l|l|l|}
\hline $\mathrm{P}(0)=1$ & $\mathrm{q}(0)=0$ & $q^{(5)}(0)=120$ \\
\hline$p^{(1)}(0)=i$ & $q^{(1)}(0)=0$ & $q^{(6)}(0)=0$ \\
\hline$p^{(2)}(0)=-1$ & $q^{(2)}(0)=0$ & \\
\hline$p^{(3)}(0)=-i$ & $q^{(3)}(0)=0$ & \\
\hline$p^{(4)}(0)=1$ & $q^{(4)}(0)=0$ & \\
\hline
\end{tabular}

Table 4.2

Then :

$\operatorname{Re} s(f, 0)=5 \times\left[\frac{1}{120}\right]=\frac{1}{24} \quad$ we get from that $\int_{0}^{\infty} \frac{\sin (x)}{x^{5}} d x=\frac{\pi}{48}$

Then by using the new procedure of order $(\mathrm{m})$ only we find general solution.

$\int_{0}^{\infty} \frac{\sin (x)}{x^{m}} d x=\left\{\begin{array}{lc}(-1)^{n+1} \frac{\pi}{2(m-1) !} & \text { if } \\ 0 & m=1,3,5,7,9 \ldots \& n=1,2,3, \ldots \\ \text { otherwise }\end{array}\right.$

\section{Example (4.4)}

Evaluate the following improper integral $\int_{0}^{\infty} \frac{\cos (x)}{x^{m}} d x$ Where $\mathrm{m}=2,4,6, \ldots$

\section{Solution:}

By the same way of example(3) we get the general solution .

$$
\int_{0}^{\infty} \frac{\cos (x)}{x^{m}} d x= \begin{cases}(-1)^{n} \frac{\pi}{2(m-1) !} & \text { if } \quad m=2,4, \ldots \& n=1,2,3 \ldots \\ 0 & \text { otherwise }\end{cases}
$$

By using the new procedure of order $(\mathrm{m})$ only we get the above solution.

\section{5-conclusions :}

It is clear that the new procedure for calculating poles of any order $(\mathrm{m})$ is a very effective rule in determining the values of improper integrals while in the most of complex variable methods fail to compute these integrations. 



\section{REFERENCES}

[1] Trott, M. , (1997) "Visualization of Riemann Surfaces of Algebraic functions", Mathematica in Education and Research, Vol.(6), no. 4, pp.3-15.

[2] Churchill, R. , (1974)" Complex Variable and Applications ", New York .

[3] Rinehart and Winston , (1976) " Elements of Complex Variable " ,Macmilan \& Co LTD London.

[4] Dauglas, N ., (2000)" complex Analysis " .

[5] Murray, R. , (1981) " Theory and problems of Complex Variable", Ginn \& Company, Boston.

[6] Oliver, K. , (1996) " The Residue theorem and it's Application_". New York .

[7] Chen, L. (2003), " Introduction to complex analysis ".

[8] Mike, P. (2005), " Complex Number and Complex Function ". New York .

[9] Vitally, M. (2005)" Complex Function theory" , University of Bristol.

[10] Sean, M. (2001) " Introduction to Applied mathematics ".

[11] Risch, R., (1979) "Algebraic Properties of the Elementary Functions of Analysis", American Journal of Mathematics, Vol.(101), pp. 743-759. 